\title{
ARTICLE OPEN Transition from source- to stress-controlled plasticity in nanotwinned materials below a softening temperature
}

\author{
Seyedeh Mohadeseh Taheri Mousavi ${ }^{1,2}$, Haofei Zhou ${ }^{1}{ }^{1}$, Guijin Zou ${ }^{1}$ and Huajian Gao ${ }^{1}$
}

Nanotwinned materials have been widely studied as a promising class of nanostructured materials that exhibit an exceptional combination of high strength, good ductility, large fracture toughness, remarkable fatigue resistance, and creep stability. Recently, an apparent controversy has emerged with respect to how the strength of nanotwinned materials varies as the twin thickness is reduced. While a transition from hardening to softening was observed in nanotwinned $\mathrm{Cu}$ when the twin thickness is reduced below a critical value, continuous hardening was reported in nanotwinned ceramics and nanotwinned diamond. Here, by conducting atomistic simulations and developing a theoretical modeling of nanotwinned Pd and Cu systems, we discovered that there exists a softening temperature, below which the material hardens continuously as the twin thickness is reduced (as in nanotwinned ceramics and diamond), while above which the strength first increases and then decreases, exhibiting a maximum strength and a hardening to softening transition at a critical twin thickness (as in nanotwinned $\mathrm{Cu}$ ). This important phenomenon has been attributed to a transition from source- to stress-controlled plasticity below the softening temperature, and suggests that different hardening behaviors may exist even in the same nanotwinned material depending on the temperature and that at a given temperature, different materials could exhibit different hardening behaviors depending on their softening temperature.

npj Computational Materials (2019)5:2 ; https://doi.org/10.1038/s41524-018-0140-5

\section{INTRODUCTION}

It has been widely demonstrated that the incorporation of twin boundaries (TBs) in the grains of nano- or ultrafine-grained materials results in significant improvement of their mechanical and physical properties. ${ }^{1-21}$ In addition to nt-metals, nanoscale TBs have also been synthesized into the crystalline lattices of $\mathrm{CBN}^{3}$ and diamond, ${ }^{4}$ with report that nt-diamond exhibits unprecedented hardness (with Vickers hardness exceeding $200 \mathrm{GPa}$ ), ultrahigh chemical stability (with oxidation temperature above $1000^{\circ} \mathrm{C}$ ), and fracture toughness above $10 \mathrm{MPa} \sqrt{\mathrm{m}}$. Interestingly, while nt-Cu exhibits a softening behavior as the twin thickness is reduced to below a critical value $\lambda_{\text {crit }} \simeq 15 \mathrm{~nm},{ }^{1,2,6,22} \mathrm{nt}-\mathrm{cBN}$ and $\mathrm{nt}-$ diamond were reported to harden continuously with reduced TB spacing. ${ }^{3,4}$ In nt-Cu, the flow stress follows the classical Hall-Petch behavior above $\lambda_{\text {crit }}$ as the TBs block the motion of dislocations similar to grain boundaries (GBs) in nanocrystalline materials, while dislocation nucleation controlled by sources at TB-GB intersections leads to a softening behavior below $\lambda_{\text {crit. }}{ }^{2}$

The reported continuous strengthening in nt-cBN and ntdiamond to unprecedented hardness values at very small TB spacing has generated significant interest in revealing the underlying deformation mechanisms, as well as a number of apparent controversies in the last few years. It was shown that the measured hardness of nanocrystalline $\mathrm{CBN}$ can depend sensitively on the measurement method: while the Vickers hardness of the material significantly increased as the grain size is reduced, the Knoop hardness remained nearly unchanged, ${ }^{23,24}$ thereby casting significant doubt on the accuracy of the measured Vickers hardness in nt-cBN and nt-diamond. ${ }^{3,4}$ On the other hand, DFT simulations and experimental observations on the structure of nanotwins in nt-cBN have provided some theoretical support for the observed hardening behavior, ${ }^{5,25-27}$ although none of the theoretical studies have directly considered realistic polycrystalline samples with embedded TBs.

Here, we demonstrate the existence of a softening temperature $T_{\mathrm{s}}$ for nt-materials, below which the material hardens continuously as the twin thickness is reduced, while above which the strength first increases and then decreases, exhibiting a maximum strength and a hardening to softening transition at a critical twin thickness. The basic phenomenon is established through molecular dynamics (MD) simulations of polycrystalline $\mathrm{nt}-\mathrm{Pd}^{28-31}$ and nt$\mathrm{Cu}^{1,2}$ samples, and by theoretical modeling that is not subjected to the usual limitations of $M D$ in size and time scales. The simulation and modeling results reveal that at very small twin thicknesses $\left(<\lambda_{\text {crit }}\right)$, the deformation is governed by the migration of TBs associated with twinning partial dislocations nucleated at TB-GB intersections. While the nucleation of twinning partials is limited by the number of dislocation sources above $T_{\mathrm{s}}$ (ref. ${ }^{2}$ ), below $T_{\mathrm{s}}$ the same nucleation process becomes limited by local stress concentration at the TB-GB intersections, whose peak stress level, decreases with reduced TB spacing, leading to continuous hardening. Thus, the softening temperature $T_{\mathrm{s}}$ demarcates a transition from source- to stress-controlled TBs migration. For a material with small $T_{\mathrm{s}}$ such as $\mathrm{nt}-\mathrm{Cu}$, source-controlled TB migration dominates over a wide range of temperatures, in which the strength of the material first increases and then decreases, exhibiting a maximum strength and a hardening to softening transition at a critical twin thickness. For materials with large $T_{\mathrm{s}}$ stress-controlled TB migration prevails over a wide range of

\footnotetext{
${ }^{1}$ School of Engineering, Brown University, Providence, RI 02912, USA and ${ }^{2}$ Department of Materials Science and Engineering, Massachusetts Institute of Technology, 77 Massachusetts Avenue, Cambridge, MA 02139, USA

Correspondence: Huajian Gao (huajian_gao@brown.edu)
}

Received: 26 July 2018 Accepted: 9 December 2018

Published online: 04 January 2019 
(a)

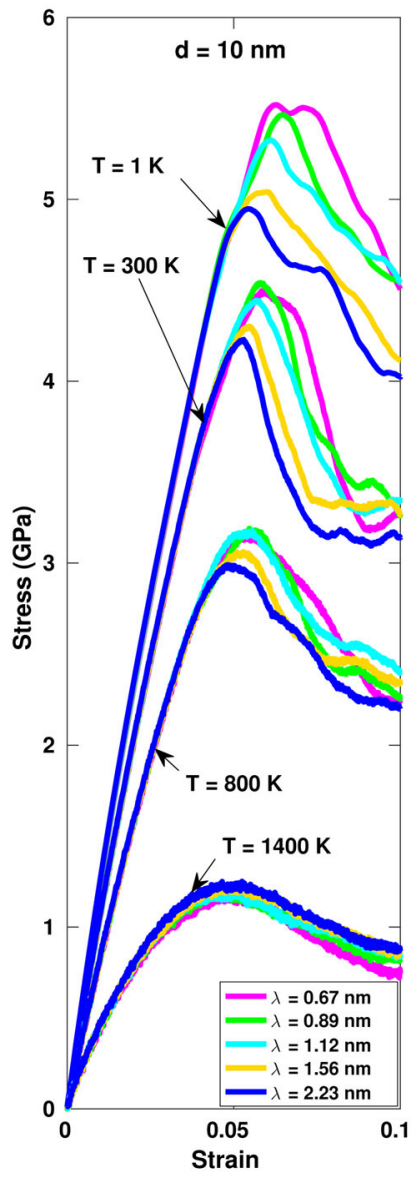

(b)
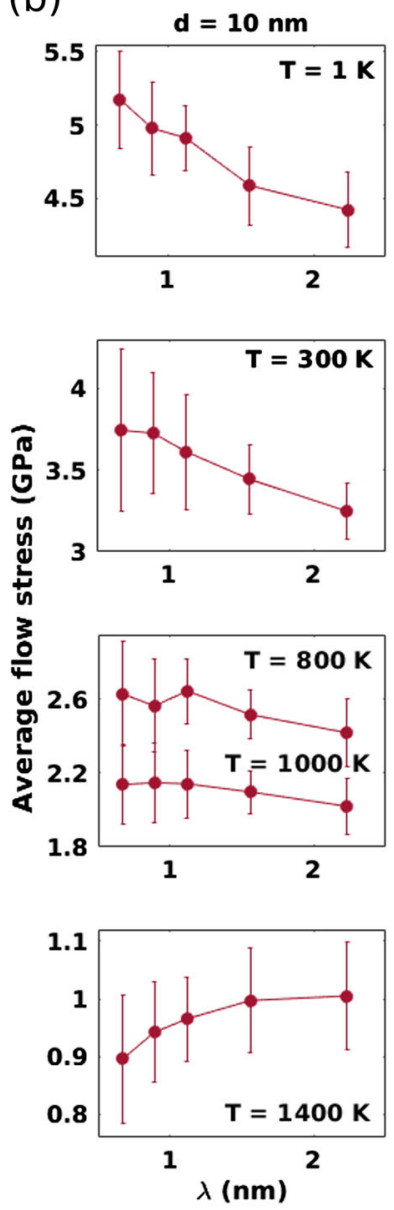

(c)

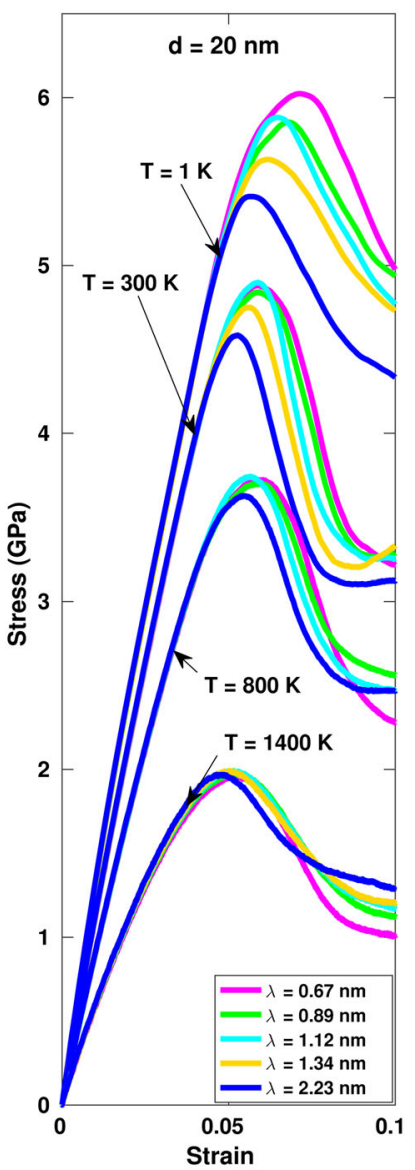

(d)
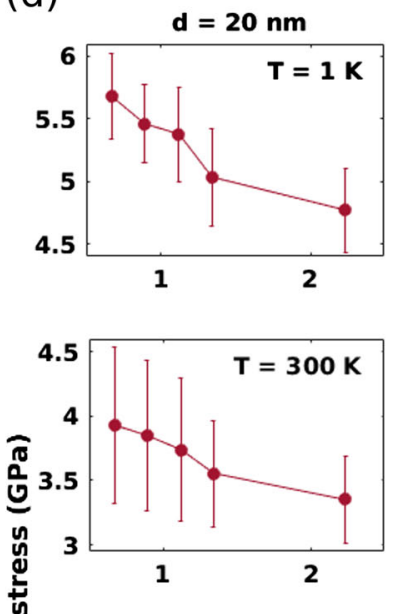

3.5
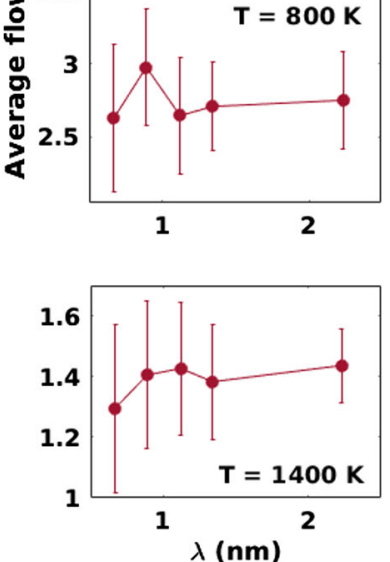

Fig. 1 Stress-strain curves and average flow stress from molecular dynamics simulations of nanotwinned Pd. a, c Simulated stress-strain relations of nanotwinned $\mathrm{Pd}$ samples with grain sizes of a $d=10 \mathrm{~nm}$ and $\mathrm{c} 20 \mathrm{~nm}$, and twin thickness varying from $\lambda=0.67$ to $2.23 \mathrm{~nm}$ at different temperatures $T=1,300,800$, and $1400 \mathrm{~K}$. b. d Average flow stress of nanotwinned Pd samples with grain sizes of $\mathbf{b} d=10 \mathrm{~nm}$ and $\mathbf{d}$ $20 \mathrm{~nm}$ at different temperatures. The average flow stress is calculated for strains ranging from 6 to $10 \%$ for each sample. A transition from hardening to softening behavior is observed by increasing the temperature. The average flow stress increases continuously by decreasing the twin thickness at lower temperatures 1 and $300 \mathrm{~K}$, while it decreases at $1400 \mathrm{~K}$ when the twin thickness is reduced. Error bars represent the standard deviation from statistical analyses

temperatures, in which continuous hardening with reduced TBs spacing would be expected.

\section{RESULTS AND DISCUSSION}

We began by simulating the tensile stress-strain behaviors of three-dimensional polycrystalline nt-Pd samples with a high density of nanoscale twins embedded in grains with sizes selected to be 10 and $20 \mathrm{~nm}$. The TB spacing varies from 0.67 to $2.23 \mathrm{~nm}$. The simulation details are provided in Methods. The tensile stress-strain curves obtained at 1, 300, 800, and $1400 \mathrm{~K}$ are plotted in Fig. 1a, c for the two grain sizes and all twin thicknesses. Figure $1 \mathrm{~b}$, d show the calculated average flow stress of the samples in the strain range of $6-10 \%$. It is seen that the average flow stress increases progressively with decreasing TB spacing at 1 and $300 \mathrm{~K}$, exhibiting a qualitatively similar trend to the experimental observation of continuous hardening behavior in nt-cBN and ntdiamond. ${ }^{3,4}$ However, a softening behavior is observed at $1400 \mathrm{~K}$, indicating a transition from hardening to softening behavior above a softening temperature $T_{\mathrm{s}}$. A comparison between the hardening behaviors at 1 and $300 \mathrm{~K}$ for both grain sizes indicates that the flow stress increases more at lower temperature, i.e., the lower the temperatures, the more the material hardens with reduced TB spacing.
To investigate the generality of the simulation results for nt-Pd, we further simulated the tensile stress-strain behaviors of polycrystalline nt-Cu samples with the same grain structure, twin boundary orientation, and boundary conditions as our nt-Pd samples, and a grain size of $10 \mathrm{~nm}$ at 1 and $300 \mathrm{~K}$ (see Fig. 2). The simulation details are provided in Methods. As shown in Fig. 2, in contrast to the previously reported room temperature softening behavior of nt-Cu below a critical TB spacing, ${ }^{1,2}$ at $1 \mathrm{~K}$ the simulated nt-Cu samples harden continuously with reduced twin thickness. This result suggests that there is also a softening temperature for $\mathrm{nt}-\mathrm{Cu}$, albeit with a smaller value than that of nt$\mathrm{Pd}$. The simulations were repeated for a different grain structure and applied strain rate (see Supplementary Discussion 1). The similar captured trend confirms the insensitivity of the results to the grain geometry and the loading rate. Moreover, our result is consistent with a recent study of $\mathrm{nt}-\mathrm{Cu}$ nanorods under torsional loading, where a continuous hardening behavior with reduced TB spacing at $1 \mathrm{~K}$ was reported. ${ }^{32}$

Further investigation was focused on the origin of the continuous hardening behavior observed in nt-Pd samples at 1 and $300 \mathrm{~K}$. Deformation patterns of a sample with grain size of $20 \mathrm{~nm}$ and two twin thicknesses $\lambda=0.67$ and $2.23 \mathrm{~nm}$ are presented in Supplementary Figure 2 for the two temperatures (see also Supplementary Movies 1-4). In all samples, we observed 
(a)

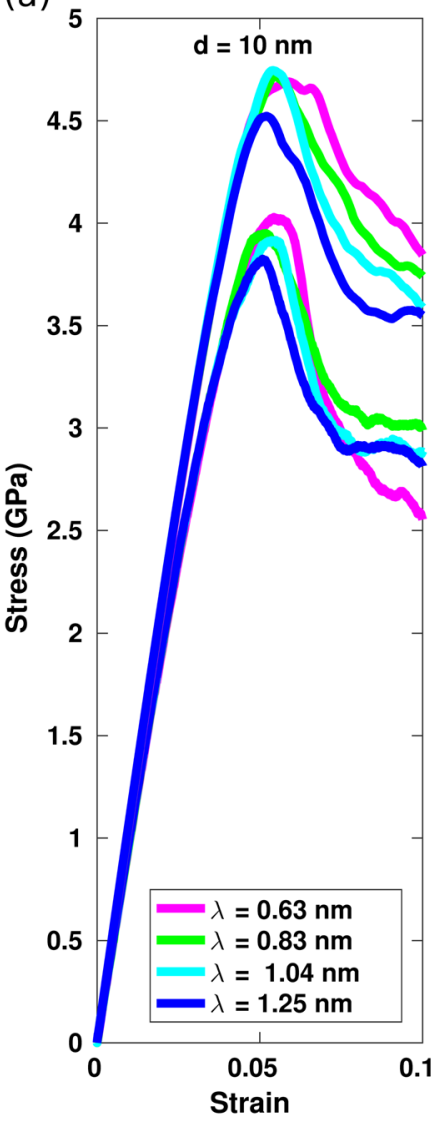

(b)

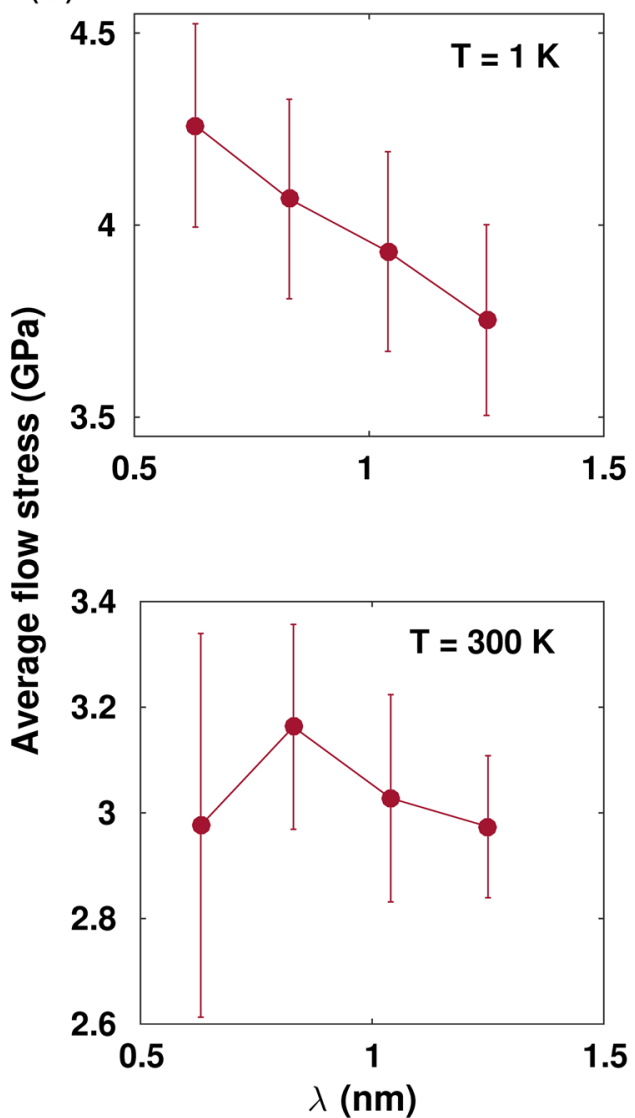

Fig. 2 Stress-strain relations and average flow stress from molecular dynamics simulations of nanotwinned polycrystalline Cu samples. a Simulated stress-strain curves of nanotwinned Cu samples with a grain size of $10 \mathrm{~nm}$ and different twin thicknesses at two temperatures $T=1$ and $300 \mathrm{~K}$. b Average flow stress calculated for the strains in the range of $6-10 \%$ as a function of the twin thickness for the simulated nanotwinned Cu samples. Continuous hardening by decreasing the twin thickness is observed at $1 \mathrm{~K}$, while at $300 \mathrm{~K}$, the flow stress first increases and then decreases below a critical twin thickness. Error bars represent the standard deviation from statistical analyses

(a)

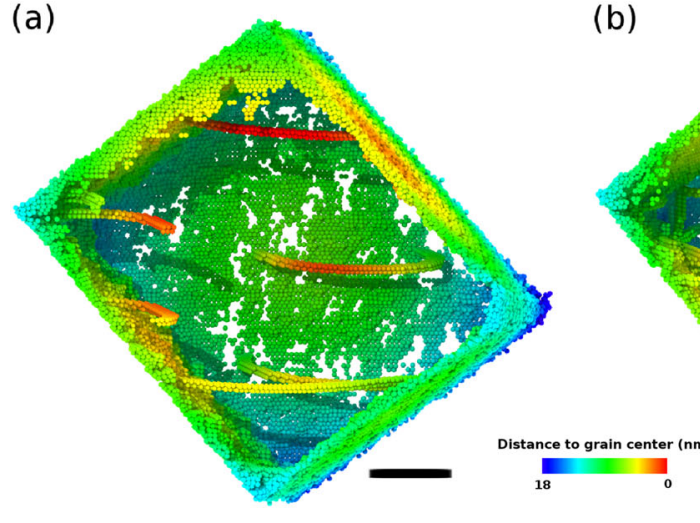

(b)

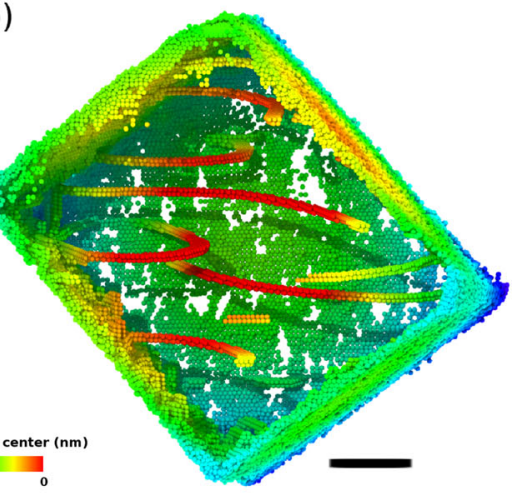

Fig. 3 Typical dislocation structures in a nanotwinned grain with a grain size of $20 \mathrm{~nm}$ at the strain of $7.6 \%$ and temperature $T=1 \mathrm{~K}$. $\mathbf{a}$, $\mathbf{b}$ Shockley partial dislocations are emitted from grain boundary-twin intersections and move along the twin boundaries in the interior of the grain for both simulated twin thicknesses $\mathbf{a} \lambda=0.67 \mathrm{~nm}$ and $\mathbf{b} 2.23 \mathrm{~nm}$. Coloring scheme applied here is based on the distance to the grain center for each atom (scale bars: $5 \mathrm{~nm}$ )

plastic deformation associated with considerable TB migrations via the gliding of Shockley partial dislocations emitted from the $T B-G B$ intersections, leading to changes in TB spacing and even detwinning inside the grains. In samples with the larger TB spacing $(\lambda=2.23 \mathrm{~nm})$, dislocations intersecting with TBs also play a role in accommodating plastic deformation. Dislocation structures in a grain with twin thicknesses $\lambda=0.67$ and $2.23 \mathrm{~nm}$ at the strain of $7.6 \%$ and $T=1 \mathrm{~K}$ are shown in Fig. $3 \mathrm{a}, \mathrm{b}$, respectively. This grain is chosen as inclined dislocations are rarely observed during its deformation (see Supplementary Movies 5 and 6). In both cases, a large amount of twinning partial dislocations moving parallel to the TBs are observed in the interior of the grain. The same observation was made at $T=300 \mathrm{~K}$ in the same grain (see Supplementary Movies 7 and 8). The total length of partial dislocations is presented as a function of the applied strain in Supplementary Figures $3 a$ and b. At both $T=1$ 
(a)

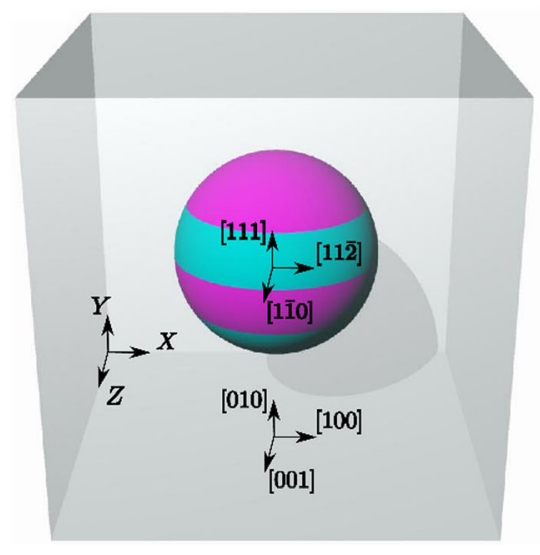

(c)

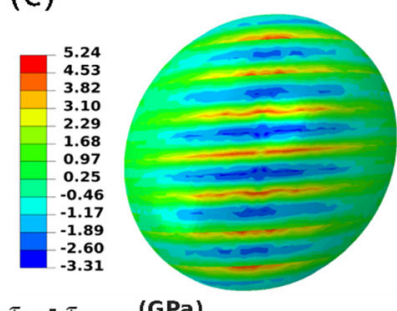

(d)

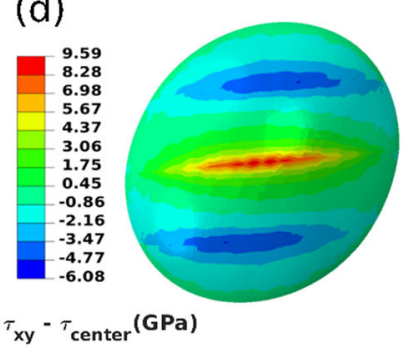

(b)

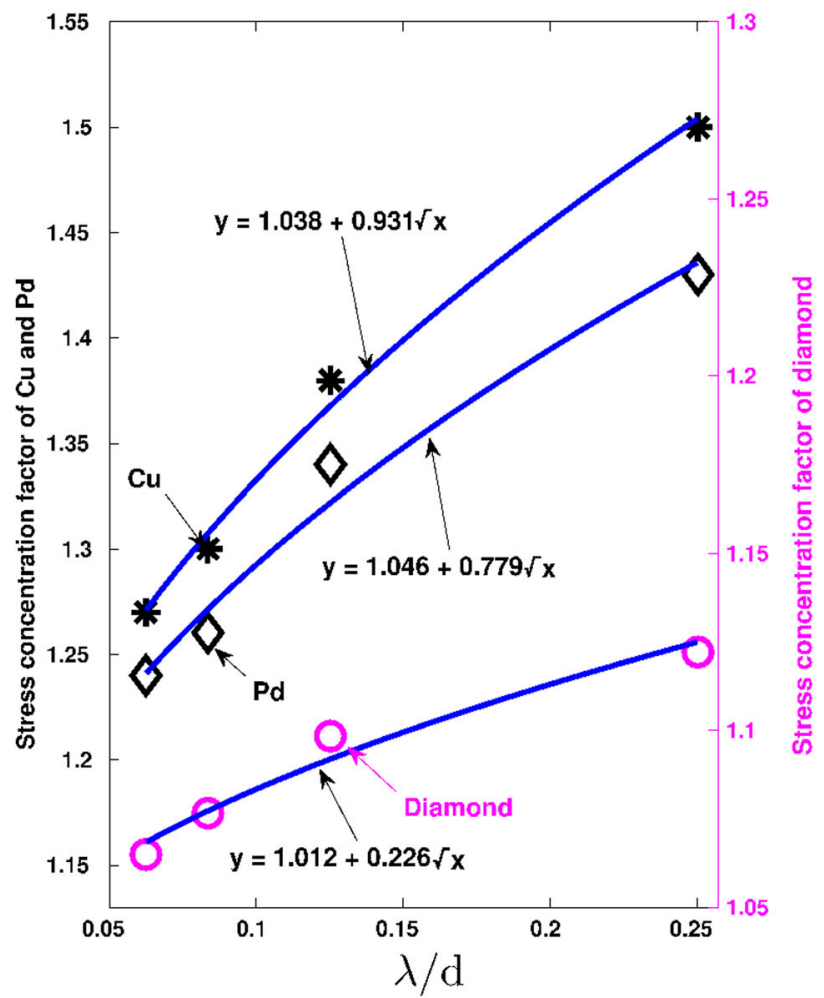

Fig. 4 Finite element simulations showing the dependency of peak stress level at grain boundary-twin intersections on twin thickness. a Finite element model of a spherical nanotwinned grain containing alternative twins (colored in blue) and matrixes (colored in purple) located at the center of a cube (colored in gray). The cube edge is two times larger than the grain diameter. The crystal orientation of the twins and the cube are indicated in the model. $\mathbf{b}$ Calculated stress concentration factors, defined as the maximum value of the resolved shear stress occurring at the grain boundary-twin intersections normalized by its value at the center of the nanotwinned grain, increases with the twin thickness for three nanotwinned materials $\mathrm{Pd}, \mathrm{Cu}$, and diamond. The stress concentration factor versus twin thickness relations for each material is fitted to a square root scaling law. The direction of the applied displacement has an inclination angle of $30^{\circ}$ with respect to the $X$ axis. c, d Contours showing the resolved shear stress along the direction on (111) planes subtracted by its value at the center of the grain for two different normalized twin thicknesses $\mathbf{c} \lambda / d=0.083$ and $\mathbf{d} 0.25$. The maximum resolved shear stress at the intersections is higher for the larger twin thickness

and $300 \mathrm{~K}$, TB migration occurs more readily with a larger average length of partial dislocations at the larger twin thickness $(\lambda=$ $2.23 \mathrm{~nm}$, see Supplementary Figure $3 \mathrm{c}$ ). The difference becomes negligible at higher temperatures for strains less than $7 \%$. These results are at odds with previous simulations of nt-Cu at the room temperature where the nucleation of twinning partial dislocations is governed by the density of nucleation sources, leading to softening with easier TB migration at smaller TB spacing. ${ }^{2}$

To explore the origin of enhanced TB migration in grains containing larger TB spacings, contours of shear stress along the slip direction on (111) planes, at the strain of $3.8 \%$ and $T=1 \mathrm{~K}$, are compared in Supplementary Figures $3 e$ and $g$ for $\lambda=0.67$ and $2.23 \mathrm{~nm}$. It can be seen that at TB-GB intersections, the shear stress reaches a higher magnitude in the nt-grain with larger TB spacing. This is in fact consistent with recent experimental observations of higher local stress at TB-GB intersections in polycrystalline $\mathrm{Cu}$ samples with larger TB spacings via transmission electron microscopy. ${ }^{33}$ We further confirmed this in thin $\mathrm{Cu}$ plates containing slanted TBs (with an inclination angle of $\simeq 19.5^{\circ}$ ) under tension at $T=1 \mathrm{~K}$ (see Supplementary Figure $4 \mathrm{a}$ and Supplementary Discussion $1 \mathrm{~b}$ ), where the strength is controlled by nucleation of twinning partials from the intersections between the free surface and the TBs (see Supplementary Movies 9 and 10). Supplementary Figure $4 \mathrm{~b}$ shows a clear hardening trend with reduced TB spacing at $T=1 \mathrm{~K}$, in distinct contrast with the softening behavior of the material at $T=600 \mathrm{~K}$ (see Supplementary Figures $4 \mathrm{f}$ and $\mathrm{g}$ ), and also the previous observation of a softening behavior in nt-Cu pillars at $300 \mathrm{~K}$ (ref. ${ }^{6}$ ). To analyze the shear stress at surface-TB intersections, we focused on the atoms surrounding the surface-TB intersections within a cutoff radius of $0.7 \mathrm{~nm}$ (marked in color green in Supplementary Figure 4c). Supplementary Figures $4 \mathrm{~d}$ and e plot the cumulative density function (CDF) of the stress component $\tau_{x y}$ of these atoms. It is seen that the strength of the sample is correlated with the stress magnitude of the atoms which only count for $<1 \%$ of the total number of atoms in the system. It is also observed that the maximum shear stress achievable in the system is higher for plates with larger TB spacing.

The dependence of the peak stress level, on the twin thickness at the TB-GB intersections was also investigated by finite element (FE) simulations. A typical grain containing alternative twin and matrix materials is placed inside a cube with a different crystalline orientation as shown in Fig. 4a. The numerical details are provided in Supplementary Discussion 2. As can be seen in Supplementary Figure 5, for different directions of the applied loading, the normalized maximum shear stress along the slip direction on (111) planes (normalized by its value at the center of the grain) is always higher in the specimen with larger TB spacing. If we fix the loading direction at $30^{\circ}$ relative to the $X$ axis, Fig. $4 \mathrm{~b}$ demonstrates that the shear stress concentration factor increases as the number of TB-GB intersections decreases (i.e., $\lambda / d$ increases) for all the simulated materials ( $\mathrm{Pd}, \mathrm{Cu}$ and, diamond). The stress contours in Fig. 4c, d show that the resolved shear stress subtracted by its value at the center of the grain changes sign at adjacent 
(a)

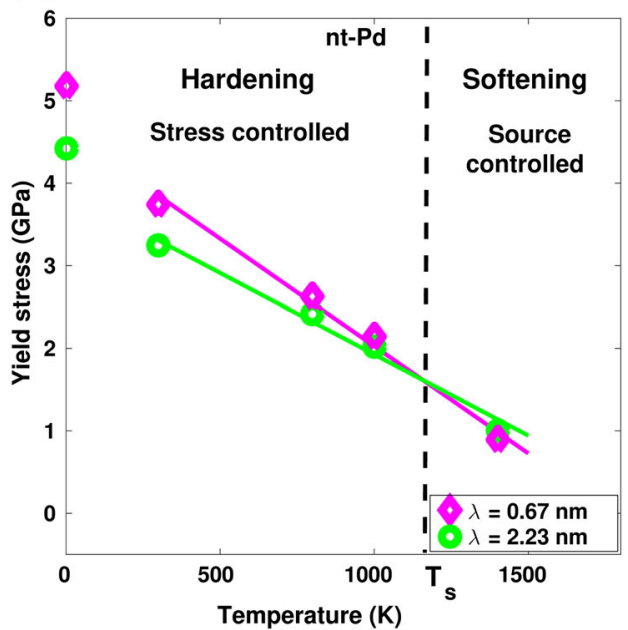

(b)

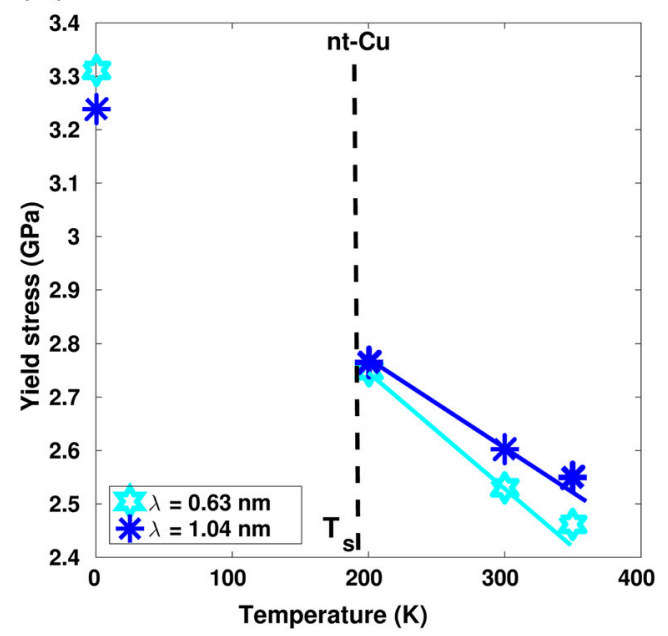

Fig. 5 a The yield stress of nanotwinned $\mathrm{Pd}(\mathrm{nt}-\mathrm{Pd})$ with a grain size of $10 \mathrm{~nm}$ as a function of the temperature. The calculated average flow stresses of nanotwinned Pd with two different twin thicknesses $\lambda=0.67$ and $2.23 \mathrm{~nm}$ along with fitting curves based on Eq. 1, revealing the existence of a softening temperature $T_{s}$, defined by the intersection between the two fitting curves. Stress-controlled hardening is revealed below $T_{s}$, while source-controlled softening is observed above $T_{s}$. b The calculated flow stresses of nanotwinned $\mathrm{Cu}$ (nt-Cu) with two different twin thicknesses $\lambda=0.63$ and $1.04 \mathrm{~nm}$ along with the theoretical fitting curves. Nanotwinned Cu has lower $T_{\mathrm{s}}$ as compared to nanotwinned Pd

intersections. The interaction between the elastic fields at adjacent TB-GB intersections reduces the peak stress level, and the closer the intersections, the larger the stress reduction. Further discussions on the relation between the stress concentration factor and TB spacing for different materials are provided in Supplementary Discussion 2.

Our simulations have demonstrated that, below a softening temperature $T_{\mathrm{s}}$, both nt-Pd and nt-Cu exhibit continuous hardening with reduced TB spacing, as the nucleation of twinning partial dislocations that govern the TB migrations becomes controlled by the stress concentration at the TB-GB intersections rather than by the number of dislocation sources. To establish the generality of these observations, it will be necessary to develop a theoretical model which is not subject to the usual limitations of MD in size and time scales. For this purpose, we adopt a kinetic theory to predict the temperature induced transition from sourceto stress-controlled nucleation of twinning partials. Details of the theoretical derivations and calculations for $\mathrm{nt}-\mathrm{Pd}$ and $\mathrm{nt}-\mathrm{Cu}$ have been provided in Supplementary Discussion 3. Based on our model, the strength of the nt-material is given by a competition between stress- and source-controlled terms as follows:

$\tau=\frac{\nabla U}{S_{\lambda} V^{*}}-\frac{k_{\mathrm{B}} T}{S_{\lambda} V^{*}} \ln \left(\alpha \beta \frac{d}{\lambda} \frac{\nu_{\mathrm{D}}}{\dot{\varepsilon}}\right)$

where $\nabla U$ is the activation energy for the twinning partial dislocation, $V^{*}$ the activation volume, $k_{\mathrm{B}}$ the Boltzmann constant, $T$ the temperature, $a$ the percentage of efficient sources accounting for GB structure/geometry, $\beta$ the Taylor factor accounting for the contribution from all possible emission systems in the thermal part, $v_{D}$ and $\dot{\varepsilon}$ the Debye frequency and applied strain rate, respectively; $S_{\lambda}$ is a factor representing the local stress concentration at the TB-GB intersections. Based on our simulations, $S_{\lambda}$ increases as the TB spacing increases, leading to a reduction in the stress-controlled term in Eq. 1. Using nudged elastic band (NEB) method, $^{34,35}$ the lower energy barrier for partial dislocation emission from GB-TB intersection of a sample with larger twin boundary spacing can be also confirmed (see Supplementary Discussion 6 and Supplementary Figure 9). The predicted flow stress based on Eq. 1 is plotted as a function of temperature in Fig. 5 a for nt-Pd samples with $d=10 \mathrm{~nm}$ at two twin thickness of $\lambda=$ 0.67 and $2.23 \mathrm{~nm}$. It is seen that the predictions at two twin thicknesses intersect at a critical temperature, defined as the softening temperature $T_{\mathrm{s}}$. Below $T_{\mathrm{s}}$ the stress-controlled term dominates and the material exhibits continuous hardening which is well captured by our model. On the other hand, at temperatures above $T_{s}$, dislocation nucleation is governed by the number of sources at TB-GB intersections, which is also captured by our model as a larger tangent of the fitted line for the sample with lower TB spacing.

Our theoretical model in Eq. 1 can also clarify the sensitivity of the softening temperature $T_{\mathrm{s}}$ to the grain growth and GB reconstruction during annealing of the sample. As explained, dislocation nucleation is governed by the local stress concentration at GB-TB intersections below the softening temperature, and this process is controlled by TB spacing rather than the grain size (see the stress-controlled term in Eq. 1). Experiments on $\mathrm{nt}-\mathrm{Cu}$ and nt-steel ${ }^{36-38}$ revealed that even after annealing of the sample at $0.5-0.8$ of the melting point, no significant changes were seen for the twin lamellae and the TB spacing remained unchanged. This is also consistent with the stability of the TBs in our MD simulations of nt-Pd samples at 1 and $300 \mathrm{~K}$ (see Supplementary Figure 2 and Supplementary Movies 1-4). Therefore, the continuous hardening behavior at extremely low temperatures does not depend on grain size and GB structure. At temperatures higher than the softening temperature, grain size and the number of efficient nucleation sources have direct influence on the source-controlled term and thus the softening behavior of the material. For a simple estimation, we assume that the grain size grows 10 times during annealing, ${ }^{38}$ and $G B$ reconstruction occurs by increasing the number of efficient nucleation sources $a$ by a factor of five. Our theoretical model predicts a softening temperature within $10 \%$ of the original value (see Supplementary Figure 6 and Supplementary Discussion 4 for more details). Therefore, our theoretical model in Eq. 1 indicates that the softening temperature is insensitive to the grain size and GB structure.

The magnitude of $T_{\mathrm{s}}$ increases with the activation energy of twinning partial dislocations and therefore should be higher for materials with stronger bonding, in consistency with our MD simulations on nt-Pd and nt-Cu. The migration of TBs is also known to dominate plastic deformation in materials with covalent bonding, ${ }^{25-27}$ and the nucleation stresses in ceramics and diamond are considerably higher than those in metals. For instance, about 20 times of increase in the activation energy was reported for $\mathrm{CBN}$ compared to face-centered cubic (fcc) metals. ${ }^{25}$ 
Therefore, it is likely that the softening temperatures for covalent and ionic materials are higher than the room temperature, similar to the case of nt-Pd, and consequently continuous hardening is observed for these materials. ${ }^{3,4}$

In conclusion, we have demonstrated that there exists a softening temperature for nt-materials, below which the material hardens continuously as the twin thickness is reduced, while above which the strength first increases and then decreases, exhibiting a maximum strength and a hardening to softening transition at a critical twin thickness. Our simulations revealed that the continuous hardening is driven by stress concentration at the TB-GB intersections, which decreases with reduced TB spacing. Our theoretical model suggests that the stronger the atomic bonding, the higher the softening temperature, and that the observed behavior could be generic to all nt-materials.

\section{METHODS}

\section{Molecular dynamics simulations}

Three-dimensional polycrystalline nt-Pd samples with dimensions of $30 \times$ $30 \times 30 \mathrm{~nm}^{3}$ and $60 \times 60 \times 60 \mathrm{~nm}^{3}$ were prepared for the MD simulations. Each sample contains 27 randomly oriented grains with mean grain sizes of $d=10$ and $20 \mathrm{~nm}$, each grain further contains a number of twin interfaces. Periodic boundary conditions are imposed at the boundaries of the samples. The grain structure and relative twin inclinations are fixed for the selected grain sizes, and the system contains $\sim 1,800,000$ atoms for $d=$ $10 \mathrm{~nm}$ and $14,500,000$ atoms for $d=20 \mathrm{~nm}$. Five different twin thicknesses $\lambda=0.67,0.89,1.12,1.56,2.23 \mathrm{~nm}$, and $\lambda=0.67,0.89,1.12,1.34,2.23 \mathrm{~nm}$ are considered in each set of simulations for $d=10$ and $20 \mathrm{~nm}$, respectively. The embedded atom method potential for $\mathrm{Pd}$ was adopted, ${ }^{39}$ and the Nose-Hoover thermostat was used to maintain an NPT ensemble. All simulations were conducted at $T=1,300,800$, and $1400 \mathrm{~K}$. The time step is set to be $2 \mathrm{fs}$. The sample was annealed for $500 \mathrm{ps}$ at $T=300,800$, and $1400 \mathrm{~K}$. In the case of $T=1 \mathrm{~K}$, the samples were first annealed at $300 \mathrm{~K}$ for $200 \mathrm{ps}$, quenched down to $1 \mathrm{~K}$ in $200 \mathrm{ps}$, and equilibrated for another $200 \mathrm{ps}$ at $1 \mathrm{~K}$ prior to straining. A total tensile strain of $10 \%$ was applied on the samples at a constant strain rate of $10^{9} \mathrm{~s}^{-1}$.

For polycrystalline nt-Cu samples, the grain structure, twin boundary orientation, and boundary conditions are identical to our nt-Pd samples. The grain size is $10 \mathrm{~nm}$ and the simulatios were conducted at 1 and $300 \mathrm{~K}$. Each nt-Cu sample contains about 2,300,000 atoms. The twin thickness varies from 0.63 to $1.25 \mathrm{~nm}\left(\right.$ ref. $^{2}$ ). The embedded atom method potential for $\mathrm{Cu}$ was adopted. ${ }^{40}$

The analysis and visualization of the simulation results were conducted using OVITO. ${ }^{41}$ In adopted atom coloring scheme, gray stands for fcc atoms, red for hexagonal-closed-packed (hcp) atoms, and purple for atoms in GBs or dislocation cores.

\section{DATA AVAILABILITY}

All data required to reproduce the findings during this study are included in this Manuscript and Supplementary Information.

\section{ACKNOWLEDGEMENTS}

The authors acknowledge financial support from Swiss National Science Foundation through Grant P2ELP2_162144 (to S.M.T.M.) and National Science Foundation through grant DMR-1709318 (to H.F.Z., G.J.Z. and H.J.G.). The simulations reported were performed on resources provided by the Extreme Science and Engineering Discovery Environment (XSEDE) through Grant MS090046 and the Center for Computation and Visualization (CCV) at Brown University. Helpful discussions with A. F. Bower, D. Gerbig, and X. Li are gratefully acknowledged.

\section{AUTHOR CONTRIBUTIONS}

H.J.G. conceived and designed the project. S.M.T.M., H.F.Z., and G.J.Z. performed the molecular dynamics simulations. S.M.T.M. conducted the finite element simulations. S.M.T.M., H.F.Z., and H.J.G. co-wrote the manuscript. All authors analyzed the results and contributed to the discussions.

\section{ADDITIONAL INFORMATION}

Supplementary information accompanies the paper on the npj Computational Materials website (https://doi.org/10.1038/s41524-018-0140-5).

Competing interests: The authors declare no competing interests.

Publisher's note: Springer Nature remains neutral with regard to jurisdictional claims in published maps and institutional affiliations.

\section{REFERENCES}

1. Lu, L., Chen, X., Huang, X. \& Lu, K. Revealing the maximum strength in nanotwinned copper. Science 323, 607-610 (2009).

2. Li, X., Wei, Y., Lu, L., Lu, K. \& Gao, H. Dislocation nucleation governed softening and maximum strength in nano-twinned metals. Nature 464, 877-880 (2010).

3. Tian, Y. et al. Ultrahard nanotwinned cubic boron nitride. Nature 493, 385-388 (2013).

4. Huang, Q. et al. Nanotwinned diamond with unprecedented hardness and stability. Nature 510, 251 (2014).

5. Taheri Mousavi, S., Zou, G., Zhou, H. \& Gao, H. Anisotropy governs strain stiffening in nanotwinned-materials. Nat. Commun. 9, 1586 (2018).

6. Jang, D., Li, X., Gao, H. \& Greer, J. Deformation mechanisms in nanotwinned metal nanopillars. Nat. Nanotechnol. 7, 594-601 (2012).

7. Lu, K. Stabilizing nanostructures in metals using grain and twin boundary architectures. Nat. Rev. Mater. 1, 16019 (2016).

8. Lu, L., Shen, Y., Chen, X., Qian, L. \& Lu, K. Ultrahigh strength and high electrical conductivity in copper. Science 304, 422-426 (2004).

9. Lu, K., Lu, L. \& Suresh, S. Strengthening materials by engineering coherent internal boundaries at the nanoscale. Science 324, 349-352 (2009).

10. Zhu, T., Li, J., Samanta, A., Kim, H. \& Suresh, S. Interfacial plasticity governs strain rate sensitivity and ductility in nanostructured metals. Proc. Natl Acad. Sci. USA 104, 3031-3036 (2007).

11. Wang, Y. et al. Defective twin boundaries in naotwinned metals. Nat. Mater. 12, 697-702 (2013).

12. Wei, Y. et al. Evading the strength-ductility trade-off dilemma in steel through gradient hierarchical nanotwins. Nat. Commun. 5, 3580 (2014).

13. Zhang, $X$. et al. Enhanced hardening in $\mathrm{Cu} / 330$ stainless steel multilayers by nanoscale twinning. Acta Mater. 52, 995-1002 (2004).

14. Wang, Y. Deformation twinning during nanoindentation of nanocrystalline Ta. Appl. Phys. Lett. 86, 101915 (2005).

15. Qin, Q. et al. Recoverable plasticity in penta-twinned metallic nanowires governed by dislocation nucleation and retraction. Nat. Commun. 6, 5983 (2015).

16. Li, B., Sui, M., Li, B., Ma, E. \& Mao, S. Reversible twinning in pure aluminum. Phys. Rev. Lett. 102, 205504 (2009).

17. Chen, M. et al. Deformation twinning in nanocrystalline aluminum. Science $\mathbf{3 0 0}$, 1275-1277 (2003).

18. Wang, J., Hirth, J. \& Tome, C. Twinning nucleation mechanisms in hexagonalclose-packed crystals. Acta Mater. 57, 5521-5530 (2009).

19. Shin, Y. et al. Nanotwin-governed toughening mechanism in hierarchically structured biological materials. Nat. Commun. 7, 10772 (2016).

20. Zhou, H., Li, X., Qu, S., Yang, W. \& Gao, H. A jogged dislocation governed strengthening mechanism in nanotwinned metals. Nano. Lett. 14, 5075-5080 (2014).

21. Zhou, X., Li, X. \& Chen, C. Atomistic mechanisms of fatigue in nanotwinned metals. Acta Mater. 99, 77-86 (2015).

22. Wang, J. et al. Detwinning mechanisms for growth twins in face-centered cubic metals. Acta Mater. 58, 2262-2270 (2010).

23. Sumiya, H., Ishida, Y., Arimoto, K. \& Harano, K. Real indentation hardness of nanopolycrystalline $\mathrm{CBN}$ synthesized by direct conversion sintering under HPHT. Diam. Relat. Mater. 48, 47-51 (2014).

24. Nagakubo, A., Ogi, H., Sumiya, H. \& Hirao, M. Elasticity and hardness of nanopolycrystalline boron nitrides: the apparent Hall-Petch effect. Appl. Phys. Lett. 105, 081906 (2014).

25. Zheng, S. et al. Structure and energetics of nanotwins in cubic boron nitrides. Appl. Phys. Lett. 109, 081901 (2016).

26. Li, B., Sun, H. \& Chen, C. Large indentation strain stiffening in nanotwinned cubic boron nitride. Nat. Commun. 5, 4965 (2014).

27. Li, B., Sun, H. \& Chen, C. Extreme mechanics of probing ultimate strength of nanotwinned diamond. Phys. Rev. Lett. 117, 116103 (2016).

28. Rösner, H., Markmann, J. \& Weissmüller, J. Deformation twinning in nanocrystalline pd. Philos. Mag. Lett. 84, 321-334 (2004).

29. Ivanisenko, Y. et al. Deformation mechanisms in nanocrystalline palladium at large strains. Acta Mater. 57, 3391-3401 (2009). 
30. Colla, M.-S. et al. High strength-ductility of thin nanocrystalline palladium films with nanoscale twins: on-chip testing and grain aggregate model. Acta Mater. 60 1795-1806 (2012).

31. Idrissi, H. et al. Ultrahigh strain hardening in thin palladium films with nanoscale twins. Adv. Mater. 23, 2119-2122 (2011).

32. Zhou, $\mathrm{H}$. et al. Torsional detwinning domino in nanotwinned one-dimensional nanostructures. Nano. Lett. 15, 6082-6087 (2015).

33. Lu, N., Du, K., Lu, L. \& Ye, H. Transition of dislocation nucleation induced by local stress concentration in nanotwinned copper. Nat. Commun. 6, 7648 (2015).

34. Henkelman, G., Uberuaga, B. \& Jonsson, H. A climbing image nudged elastic band method for finding saddle points and minimum energy paths. J. Chem. Phys. 113, 9901-9904 (2000).

35. Rao, S. et al. Calculations of intersection cross-slip activation energies in fcc metals using nudged elastic band method. Acta Mater. 59, 7135-7144 (2011).

36. Zhang, X. \& Misra, A. Superior thermal stability of coherent twin boundaries in nanotwinned metals. Scr. Mater. 66, 860-865 (2012).

37. Anderoglu, O., Misra, A., Wang, H. \& Zhang, X. Thermal stability of sputtered cu films with nanoscale growth twins. J. Appl. Phys. 103, 094322 (2008).

38. Zhang, X. et al. Thermal stability of sputter-deposited 330 austenitic stainlesssteel thin films with nanoscale growth twins. Appl. Phys. Lett. 87, 233116 (2005).

39. Zhou, X., Johnson, R. \& Wadley, H. Misfit-energy-increasing dislocations in vapordeposited CoFe/NiFe multilayers. Phys. Rev. B 69, 144113 (2004).
40. Mishin, Y., Mehl, M., Papaconstantopoulos, D., Voter, A. \& Kress, J. Structural stability and lattice defects in copper: Ab initio, tight-binding, and embeddedatom calculations. Phys. Rev. B 63, 224106 (2001).

41. Stukowski, A., Albe, K. \& Farkas, D. Nanotwinned fcc metals: strengthening versus softening mechanisms. Phys. Rev. B 82, 224103 (2010).

(i) Open Access This article is licensed under a Creative Commons Attribution 4.0 International License, which permits use, sharing, adaptation, distribution and reproduction in any medium or format, as long as you give appropriate credit to the original author(s) and the source, provide a link to the Creative Commons license, and indicate if changes were made. The images or other third party material in this article are included in the article's Creative Commons license, unless indicated otherwise in a credit line to the material. If material is not included in the article's Creative Commons license and your intended use is not permitted by statutory regulation or exceeds the permitted use, you will need to obtain permission directly from the copyright holder. To view a copy of this license, visit http://creativecommons. org/licenses/by/4.0/.

(c) The Author(s) 2019 